\title{
Comparison of Antibacterial Effect of New Herbal Mouth (Miswak) Wash With Different commercial Mouth Washes on Dental Biofilm From Orthodontic Ligatures -In Vitro Study
}

\author{
S.F. Hussain ${ }^{*}$, Fouad H. AL-Bayaty, N. R.Abdul Rahim, M. A. Abdulla and Hasnah bt Belgum \\ Centre of Studies for Paediatric Dentistry \& Orthodontics, Faculty of Dentistry, Universiti Teknologi MARA, 40450 \\ Shah Alam,Selangor Darul Ehsan; E-mail:drsabafouad@yahoo.com,saba639@salam.uitm.edu.my
}

Clinical study has shown that patients who get the orthodontic treatment are more susceptible for having the enamel white spot formation (Eliades et al., 1995) (Badawi et al., 2003). Since the advent of fixed orthodontic appliance for adult patients and its use has become increasingly popular, bringing about the need to address questions regarding microorganism adherence and biofilm development (Menzaghi, 1991; Lee, 2000)

Objectives: To compare the antimicrobial effects of commercially available mouth washes and new herbal mouth wash (Miswak) on dental biofilm isolated from orthodontic ligatures and to assess bacterial morphology before and after treatment with mouthwashes.

Material and method: Five commercially available mouth washes were included in this study (Oradex, Listerine, Oral B, Salviathymol N and Colgate plax), one new herbal mouth wash (Miswak aqueous and alcohol extracts) and sterilized distilled water was used as a control. Orthodontic elastic and stainless steel ligatures were collected from 25 patients after 3 week of orthodontic treatment. Bacteria isolated then the gram stain used to identify the bacteria attached to orthodontic ligatures. Antimicrobial activity assessed by using Minimal Bactericidal Concentration test (MBC) to determine the lowest concentration of bacteria inhibited by the fixed amount of tested mouthwashes after an overnight aerobic and anaerobic incubation at $37^{\circ} \mathrm{C}$. Assessment of bacteria morphology performed with Scanning Electron Microscopy (SEM) before and after treatment with mouth washes.

Results: Only cocci gram positive bacteria identified from ligature of all samples. MBC showed absence of bacteria growth in all tested mouthwashes. SEM examination of bacteria showed variable alterations in the morphology of the isolated bacteria.

Conclusion: This study showed that Miswak ethanol extract and commercially available mouth washes exhibit strong antimicrobial activity against gram positive cocci. While Oradex represents significant morphological changes comparing to other mouth washes. On the other hand Miswak aqueous extract seems to have weak antibacterial effects against all tested bacteria samples. 